\title{
The Association between Early Gram-Negative Bacteria in Tracheal Aspirate Cultures and Severe Bronchopulmonary Dysplasia among Extremely Preterm Infants Requiring Prolonged Ventilation
}

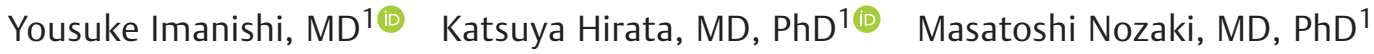 \\ Narutaka Mochizuki, MD ${ }^{1}$ Shinya Hirano, MD ${ }^{1}$ Kazuko Wada, MD ${ }^{1}$ \\ ${ }^{1}$ Department of Neonatal Medicine, Osaka Women's and Children's \\ Hospital, Izumi, Osaka, Japan \\ Am J Perinatol \\ Address for correspondence Yousuke Imanishi, MD, Department of \\ Neonatal Medicine, Osaka Women's and Children's Hospital, 840 \\ Murodo-cho, Izumi, Osaka 594-1101, Japan \\ (e-mail: rasheed0421@yahoo.co.jp).
}

Abstract

Keywords

- bronchopulmonary dysplasia

- microbiome

- Gram-negative rods

- extremely preterm infants factors associated with BPD. weeks.

Objective The study aimed to evaluate the association between bronchopulmonary dysplasia (BPD) development at 36 weeks' postmenstrual age (PMA) and Gramnegative bacteria in tracheal aspirate cultures among extremely preterm infants.

Study Design This study has a retrospective cohort. Patients were 155 infants aged less than or equal to 26 gestational weeks who were admitted to the neonatal intensive care unit of Osaka Women's and Children's Hospital from 2009 to 2018. Primary outcome was respiratory outcomes expressed as BPD development.

Multivariable logistic regression analysis was used to identify neonatal and bacterial

Results After adjusting for gestational age, birth weight, sex, chorioamnionitis, Gram-positive cocci (GPC) and Gram-negative rods (GNRs) in tracheal aspirate cultures within 28 days after birth, GNRs were significantly associated with BPD development (odds ratio [OR]: $3.88,95 \%$ confidence interval [CI]: 1.68-8.94). In contrast, GPCs were not associated with BPD development (OR: 0.47, 95\% Cl: 0.05-1.61).

Conclusion Gram-negative bacteria in tracheal cultures within 28 days of birth are associated with BPD development in infants aged less than or equal to 26 gestational

Key Points

- BPD is a factor for morbidity in extremely preterm infants.

- Respiratory infection is an adverse outcome of BPD.

- GNRs in tracheal cultures soon after birth disturb BPD development.

- GPC was not associated with BPD development.

received

November 22, 2020 accepted after revision August 5, 2021
DOI https://doi.org/ 10.1055/a-1580-3069. ISSN 0735-1631.

\footnotetext{
(C) 2021. The Author(s).

This is an open access article published by Thieme under the terms of the Creative Commons Attribution-NonDerivative-NonCommercial-License, permitting copying and reproduction so long as the original work is given appropriate credit. Contents may not be used for commercial purposes, or adapted, remixed, transformed or built upon. (https://creativecommons.org/ licenses/by-nc-nd/4.0/) Thieme Medical Publishers, Inc., 333 Seventh Avenue, 18th Floor, New York, NY 10001, USA
} 
Bronchopulmonary dysplasia (BPD) is a common complication associated with short- and long-term morbidity in extremely preterm (EP) infants. ${ }^{1,2}$ A survey of very lowbirth-weight infants performed by the Neonatal Research Network of Japan showed that the incidence of BPD remains unchanged despite advances in perinatal medicine. ${ }^{3}$

Although the mechanism of BPD development remains unclear, it is related to prematurity, inflammation, and lung injuries such as ventilator-associated lung injury and oxygen toxicity. ${ }^{4,5}$ Among these factors, lung inflammation may be the central mechanism of BPD development. Stoll et $\mathrm{al}^{6}$ reported that postnatal infections were associated with the development of neonatal complications such as BPD, severe intraventricular hemorrhage (IVH), periventricular leukomalacia, and poor growth. Among various inflammatory triggers, bacterial colonization or infection have been implicated. Proteolytic degradation of elastic fibers caused by lung infection is significant in impaired lung development in BPD. ${ }^{7}$

The respiratory tract in preterm infants was once considered sterile at birth. However, 16S rRNA genetic sequencing has shown that airways are not sterile and are colonized with diverse bacterial species within the first 72 hours of life..$^{8,9}$ Reduced diversity in the airway microbiome is associated with BPD development, leading to characterization of the pulmonary and airway microbiomes at birth. ${ }^{8,10}$ After prolonged ventilation of more than 2 weeks, the airway microbiome includes less normal flora and higher proportions of Pseudomonas aeruginosa, Klebsiella pneumoniae, and Staphylococcus aureus. ${ }^{9}$

Colonization by Gram-negative rods (GNRs) in tracheal aspirate cultures has been associated with greater BPD severity in preterm infants. ${ }^{11}$ Several observational studies showed that potentially pathogenic GNRs become more prevalent in the airway as the mechanical ventilation duration increases. ${ }^{12}$ Presence of GNRs compared with Gram-positive cocci (GPC) in bronchoalveolar lavage samples of BPD infants is associated with high-fluid levels of proinflammatory interleukins. ${ }^{13}$ The appearance of these interleukins clearly indicates inflammation, which is one cause of BPD.

However, few data exist on the association between GNRs in tracheal aspirate cultures and adverse pulmonary outcomes among BPD infants. In addition, even EP infants tend to undergo early extubation, making it difficult to collect tracheal aspirate cultures in good time. We hypothesized that GNRs in tracheal aspirate cultures within 28 days after birth are associated with BPD development in infants aged less than 26 gestational weeks.

\section{Patients and Methods}

\section{Study Design}

This retrospective cohort study included EP infants born at Osaka Women's and Children's Hospital (Osaka, Japan) who were admitted to the hospital's neonatal intensive care unit (NICU) between July, 2009 and December, 2018. Infants who had major congenital anomalies, were outborn or transferred to another hospital before discharge, died before 36 weeks' postmenstrual age (PMA), and were extubated before day 28 or never intubated were excluded. The institutional ethics committee of our center approved the study protocol (no.1430). Infants born at 27 gestational weeks of age were almost excluded, that is, they were extubated before 28 days after birth (41/58, 71\%). This study was a retrospective chart review, so informed consent was not required.

\section{Outcomes and Perinatal Factors}

The primary outcomes were BPD at 36 weeks' PMA. BPD was defined based on the requirement for oxygen or continuous positive airway pressure at 36 weeks' PMA. The outcome of supplemental oxygen use at discharge included infants who received oxygen via nasal cannula after developing hypoxia during the work of breathing and oral feeding after 37 weeks' PMA. In our institution, hypoxia episodes were defined as a persistently lower $\mathrm{SpO}_{2}$ value than the target $\mathrm{SpO}_{2}$. The target $\mathrm{SpO}_{2}$ range was maintained at 90 to $95 \%$ within the first week of life and at 88 to $95 \%$ from 1 to 36 weeks' PMA. Work of breathing is defined as an increased respiratory workload with signs of respiratory distress such as an increased respiratory frequency and retraction. After 34 weeks' PMA, if an infant's condition became stronger and the coordinated movements of breathing, sucking, and swallowing were judged to be established, oral feeding of the child was instructed.

Secondary outcomes were oxygen use at discharge, days on mechanical ventilation and supplementary oxygen during hospitalization in the neonatal intensive care unit.

Demographic factors and other clinical data were collected from the mothers' and infants' discharge summary reports and were confirmed by a detailed chart review. Small for gestational age (SGA) was defined as birth weight (BW) $\leq$ -1.5 standard deviations. For weight increase, we used the Japanese neonatal charts for gestational age at birth. ${ }^{14}$ Surfactants administered within 28 days of age were only defined as the presence or absence of the surfactant, regardless of the number of times it was administered. Symptomatic patent ductus arteriosus (PDA) requiring surgical intervention was defined as PDA with hemodynamically significant symptoms (e.g., increased pulse pressure, reduced urine output, and/or prerenal renal failure). Indomethacin or ibuprofen was used for medical treatment of symptomatic PDA. If the PDA remained symptomatic despite repeated courses of medication (i.e., $\geq 3$ courses) or if the infant exhibited complications due to medication, surgical ligation was performed. Necrotizing enterocolitis (NEC) was defined as Bell classification stage II or greater. ${ }^{15}$ Severe IVH was defined in accordance with Papile classification as grade III or IV. ${ }^{16}$ Blood transfusion was defined as a transfusion of red blood cells. Sepsis was indicated by a positive culture for bacteremia or fungemia. Diagnosing ventilator-associated pneumonia (VAP) during the neonatal period is a very difficult task. Therefore, we diagnosed VAP by the presence of fever, purulent tracheal secretions, blood leukocyte count, and abnormal shadows on chest X-rays. Cases meeting the above criteria were extracted as VAP. At our institution, we administered cefotaxime (CTX) + amikacin (AMK) for suspected sepsis, and prophylactically administered cefazolin $(\mathrm{CEZ})+\mathrm{AMK}$ for 3 days in cases where the gestational age 
(GA) was less than 26 weeks. Airway disease was defined as tracheomalacia, subglottic stenosis, or glottic stenosis, diagnosed via tracheoscopy for cases in which extubation or tracheal intubation were difficult. The duration of antibiotics use was defined as the number of days of antibiotics use prior to the first detection of any positive culture, because we generally use antibiotics to treat preterm infants of less than or equal to 26 weeks' GA.

\section{Definition of Tracheal Aspirate Cultures}

Infection surveillance for bacterial colonization is performed routinely for infants hospitalized in our neonatal intensive care unit (NICU). Tracheal aspirate cultures were obtained from birth to the last extubation once weekly. Briefly, $0.5 \mathrm{~mL}$ of sterile $0.9 \%$ saline was instilled via endotracheal tube, which followed by three to five ventilator breaths. For EP infants, a size of 5 or 6 French sterile suction catheter was inserted to $0.5 \mathrm{~cm}$ below the end of the endotracheal tube, and secretions were suctioned into an attached sterile specimen trap as the catheter was slowly withdrawn. No samples were obtained after extubation. Clinical laboratory technologists at the bacterial laboratory at Osaka Women's and Children's Hospital evaluated all samples for bacterial colonization type. Tracheal cultures were considered early-positive when they contained some microbiome at least once within 28 days after birth. GNRs were defined as K. pneumoniae, K. oxytoca, Escherichia coli, Enterobacter cloacae, Acinetobacter baumannii, P. aeruginosa, Neisseria, and Serratia marcescens. GPCs were defined as Staphylococcus spp., Group B Streptococcus, Enterococcus, Corynebacterium, and Bacillus. S. aureus was tested for methicillin susceptibility, such as methicillin-susceptible $S$. aureus and methicillin-resistant $S$. aureus (MRSA). Fungi in the tracheal cultures were Candida albicans, C. glabrata, and C. parapsilosis. We also investigated the association between the timing of GNR-positive cultures and BPD.

\section{Statistical Analysis}

A sample size of 72 patients for each study group was originally calculated to be sufficient for detecting an assumed reduction in severe BPD from 90\% in the GNR-positive group to $70 \%$ in the GNR-negative group with $80 \%$ power by a two-sided test with a 5\% significance level. Between-groups comparisons were performed on the baseline characteristics and outcomes. Fisher's exact test was used for categorical variables and the nonparametric Mann-Whitney U test was used for continuous variables. Data are presented as medians (interquartile range) or numbers (\%). Multivariable logistic regression analysis was used to identify neonatal factors associated with GNRs in tracheal cultures within 4 weeks. The potential risk factors and predictors assessed in the present study were GA, sex, SGA, antenatal steroids, and infections with GPC within 4 weeks after birth and GNRs within 4 weeks after birth. Data management and statistical analyses were performed by using $\mathrm{R}$ statistical software, version 3.4.1 ( $\mathrm{R}$ Foundation for Statistical Computing, Vienna, Austria). All reported $p$-values are two-sided; $p$ $<0.05$ was considered statistically significant.

\section{Results}

\section{Population Characteristics}

A total of 243 infants born at 22 to 26 gestational weeks were admitted to the NICU between July 1, 2009 and December 31, 2018. Of these, 155 (63.8\%) met the criteria for study inclusion. However, 88 infants were excluded because they had congenital anomalies $(n=15)$, were outborn or transferred to another hospital $(n=6)$, died within 36 weeks' PMA $(n=26)$, were not administered ventilator management $(n=1)$, or were extubated before day $28(n=66)$. The numbers of exclusion add up to more than the number of infants excluded, which is because some infants had more than one

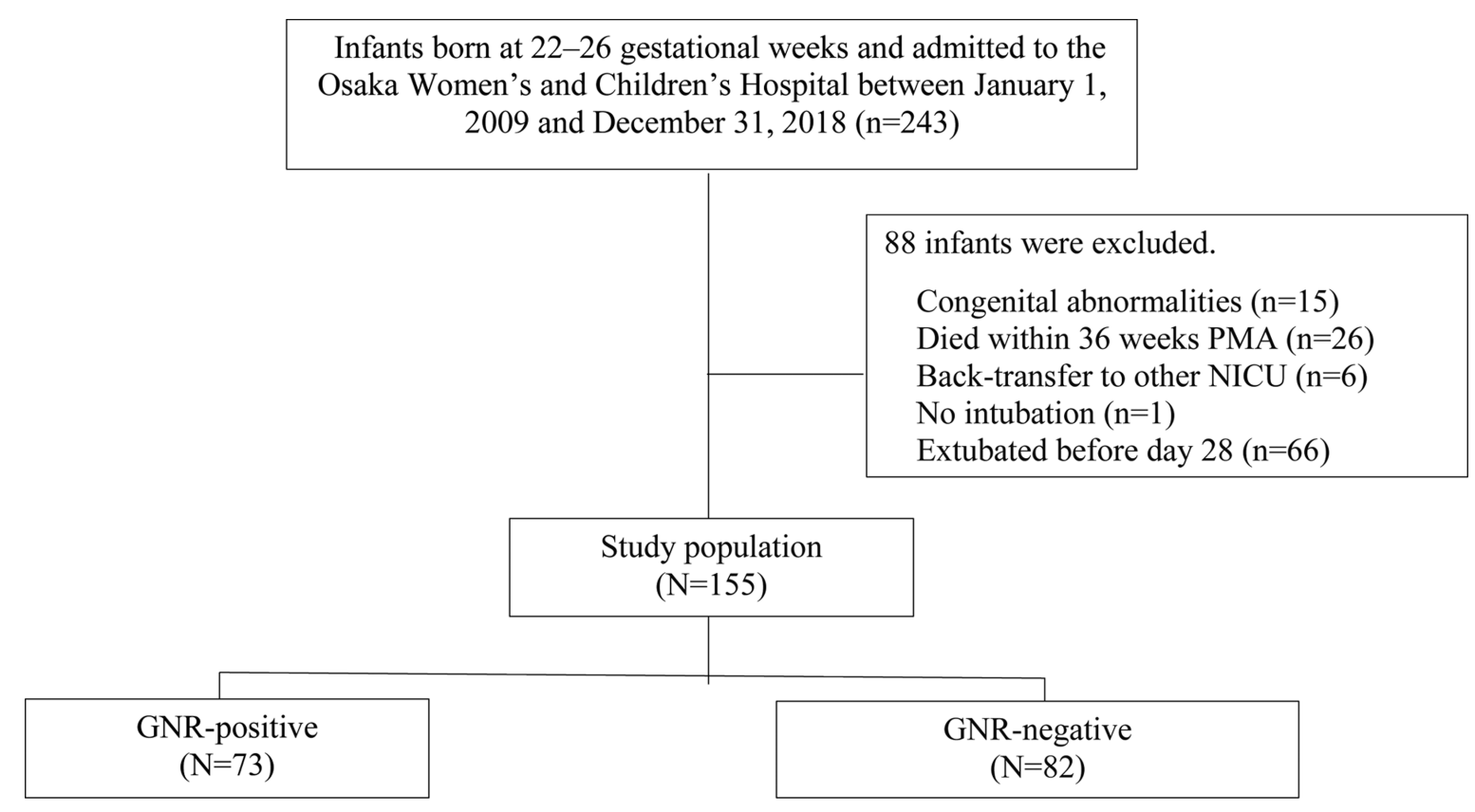

Fig. 1 Flow chart of patient enrollment. 


\begin{tabular}{|c|c|c|c|}
\hline & $\begin{array}{l}\text { GNR-positive } \\
(n=73)\end{array}$ & $\begin{array}{l}\text { GNR-negative } \\
(n=82)\end{array}$ & $p$-Value \\
\hline Gestational age, wk & $24.5(23.5-25.5)$ & $24.5(23.5-25.6)$ & 0.26 \\
\hline Birth weight, g & $615(542-743)$ & $647(550-774)$ & 0.30 \\
\hline Small for gestational age $(\leq-1.5 \mathrm{SD})$ & $11(15)$ & $13(11)$ & 0.63 \\
\hline Male & $30(41)$ & $41(50)$ & 0.19 \\
\hline Apgar's score at $1 \mathrm{~min}$ & $3.0(2-4)$ & $3.5(2-5)$ & 0.42 \\
\hline Apgar's score at $5 \mathrm{~min}$ & $6(5-8)$ & $7(5-7)$ & 0.73 \\
\hline Antenatal steroids & $53(73)$ & $62(76)$ & 0.72 \\
\hline Chorioamnionitis & $45(62)$ & $43(52)$ & 0.33 \\
\hline Surfactant use within $28 \mathrm{~d}$ after birth & $62(84)$ & $90(81)$ & 0.83 \\
\hline PDA requiring ligation & $17(23)$ & $13(16)$ & 0.31 \\
\hline NEC & $4(5)$ & $10(5)$ & 1.00 \\
\hline Severe IVH & $3(4)$ & $10(12)$ & 0.09 \\
\hline Transfusion of RBCs & $58(79)$ & $62(76)$ & 1.00 \\
\hline Sepsis & $2(3)$ & $4(5)$ & 0.43 \\
\hline VAP & $4(5)$ & $3(4)$ & 0.45 \\
\hline Fungus in tracheal culture & $7(9)$ & $7(9)$ & 0.91 \\
\hline Airway disease & $4(5)$ & $4(5)$ & 0.45 \\
\hline Postmenstrual corrected age at discharge & $39.3(37.0-43.2)$ & $41.0(39.2-43.3)$ & 0.01 \\
\hline Days using antibiotics before positive culture & $4(3-6)$ & $4(3-6)$ & 0.99 \\
\hline
\end{tabular}

Abbreviations: GNR, Gram negative rod; IVH, intraventricular hemorrhage; NEC, necrotizing enterocolitis; NICU, neonatal intensive care unit; PDA, patent ductus arteriosus; RBCs, red blood cells; VAP, ventilator associated pneumoniae.

Note: Data are expressed as the median (interquartile range) or number (\%).

reason. Of the 155 infants enrolled, $73(47.1 \%)$ were GNRpositive, and $82(52.9 \%$ ) were GNR-negative ( - Fig. 1 ). About 12 (7.7\%) were both GPC- and GNR-negative. All 155 infants were of Japanese ethnicity, and the respective median GA and BW values were 24.5 weeks (23.5-25.5 weeks) and $615 \mathrm{~g}$ (542-743 g) in the GNR-positive group and 24.5 weeks (23.525.6 weeks) and $647 \mathrm{~g}(550-774 \mathrm{~g})$ in the GNR-negative group. Perinatal factors, defined as GA, BW, sex, Apgar's scores at 1 or 5 minutes, SGA, use of antenatal steroids, history of chorioamnionitis, use of surfactant, PDA requiring ligation, incidence of NEC, severe IVH, and sepsis, did not significantly differ between the groups ( - Table 1 ).

\section{Pulmonary Outcomes}

Tracheal culture data were obtained from the laboratory once weekly and averaged five times from birth to day 28 . BPD at 36 weeks' PMA was significantly greater in the GNRpositive group than in the GNR-negative group (62/73 cases [88\%] vs. $51 / 82$ cases [62\%], $p=0.001$; - Table 2 ). Compared with the GNR-negative group, the GNR-positive group spent more days on mechanical ventilation (52 days [36-64] vs. 37 days [24-54], $p=0.04$ ) and supplementary oxygen (82 days [53-126] vs. 54 days [29-97], $p=0.01$ ), but the percentage of $\mathrm{O}_{2}$ use at discharge did not differ (31 cases [42\%] vs. 27 cases [33\%], $p=0.21$; - Table 2). These differences were not observed for infants with GPCs in their tracheal cultures (-Supplementary Table S1 and S2 [available online only]).
Regarding airway diseases, the GPC-positive group had significantly more airway lesions than did the GPC-negative group, but this was not associated with GNRs. MRSA was detected within the first month of life in all cases that exhibited airway disease. We also found no association between the timing of a positive culture and BPD (BPD: 21 days [14-28] vs. no BPD: 14 days [7-22], $p=0.11$ ). There was no association between GNR detection and the duration

Table 2 Development of bronchopulmonary dysplasia with or without Gram negative rods in the airway microbiome

\begin{tabular}{llll|} 
& $\begin{array}{l}\text { GNR-positive } \\
(\boldsymbol{n}=73)\end{array}$ & $\begin{array}{l}\text { GNR-negative } \\
(\boldsymbol{n}=\mathbf{8 2})\end{array}$ & p-Value \\
$\begin{array}{l}\text { BPD at 36 weeks' } \\
\text { PMA }\end{array}$ & $62(88)$ & $51(62)$ & 0.001 \\
$\begin{array}{l}\mathrm{O}_{2} \text { use at } \\
\text { discharge }\end{array}$ & $31(42)$ & $27(33)$ & 0.21 \\
$\begin{array}{l}\text { Days on } \\
\text { mechanical } \\
\text { ventilation (d) }\end{array}$ & $52(36-64)$ & $37(24-54)$ & 0.04 \\
$\begin{array}{l}\text { Days on } \\
\text { supplementary } \\
\text { oxygen (d) }\end{array}$ & $82(53-126)$ & $54(29-97)$ & 0.01 \\
\hline
\end{tabular}

Abbreviations: BPD, bronchopulmonary dysplasia; PMA, postmenstrual corrected age.

Note: Data are expressed as the median (interquartile range) or number (\%). 


\begin{tabular}{|c|c|c|c|c|c|c|}
\hline & & Crude & & & Adjusted & \\
\hline & Odds ratio & $95 \% \mathrm{Cl}$ & $p$-Value & Odds ratio & $95 \% \mathrm{Cl}$ & $p$-Value \\
\hline Gestational age, wk & 0.93 & $0.89-0.97$ & 0.005 & 0.93 & $0.88-0.97$ & 0.01 \\
\hline Male & 0.83 & $0.42-1.63$ & 0.58 & 1.23 & $0.56-2.71$ & 0.61 \\
\hline Small for gestational age ( $\leq-1.5 \mathrm{SD})$ & 0.45 & $0.18-1.11$ & 0.09 & 0.66 & $0.21-2.07$ & 0.48 \\
\hline Antenatal steroids & 1.42 & $0.17-5.01$ & 0.17 & 1.95 & $0.31-6.42$ & 0.11 \\
\hline GPCs within 4 wk & 0.61 & $0.16-2.34$ & 0.47 & 0.25 & $0.05-1.61$ & 0.09 \\
\hline GNRs within 4 wk & 1.79 & $1.14-2.82$ & 0.01 & 2.51 & $1.10-5.77$ & 0.03 \\
\hline
\end{tabular}

Abbreviations: Cl, confidence interval; GNR, Gram-negative rod; GPC, Gram-positive cocci; PMA, postmenstrual corrected age; SD, standard deviation.

of antibiotic administration, which amounted to 4 days (3-6) in the GNR-positive group and 4 days (3-6) in the GNRnegative group.

Univariable analysis showed that BPD at 36 weeks' PMA was significantly associated with a lower GA, and more GNRs in the tracheal cultures within 4 weeks after birth. After adjusting for GA, sex, SGA, antenatal steroids, GPCs within 4 weeks after birth and GNRs within 4 weeks after birth, BPD at 36 weeks' PMA was significantly associated with a higher frequency of GNR within 4 weeks after birth (odds ratio [OR]: $2.51,95 \%$ confidence interval [CI]: 1.10-5.77) and lower GA (OR: 0.93, 95\% CI: 0.88-0.97; - Table 3).

\section{Discussion}

In the present study with retrospective cohort, GNRs in tracheal cultures within 28 days after birth was significantly associated with BPD development in infants aged between 22 and 26 gestational weeks. Early GNRs detection was associated with BPD at 36 weeks' PMA and a longer duration of mechanical ventilator management and supplementary oxygen. These results are consistent with previous studies involving traditional covariate adjustment in regression models. ${ }^{17}$ In the full cohort from this study, GNRs in the tracheal cultures were not associated with neonatal complications such as SGA, chorioamnionitis, surfactant use, PDA, NEC, severe IVH, or sepsis. Respiratory factors were associated with GNRs but not with oxygen administration at discharge. Univariable analysis ( $\sim$ Table 1 ) showed that the corrected age at discharge was significantly later in the GNRs-positive group. This could be due to high demand for supplementary oxygen at 36 weeks' PMA.

In the present study, tests for bacteria in tracheal cultures were $92.3 \%$ positive within 4 weeks after birth among infants of less than or equal to 26 weeks' GA, which is consistent with previous reports. Cordero et al found airway colonization in over $80 \%$ of infants aged $<27$ gestational weeks who were intubated for $\geq 2$ weeks. ${ }^{18}$ Lel et al reported that the airway microbiome at birth among extremely low birthweight infants showed Firmicutes, Proteobacteria Bacteroides, Acinetobacter, and Tenericutes (including Ureaplasma spp.) on the first day of life. ${ }^{10}$ Several studies found that the
GNRs in tracheal cultures became more frequent as the mechanical ventilation duration increased. ${ }^{19}$ We also found that $P$. aeruginosa was detected within the first month of life in $6.5 \%$ of infants aged $<26$ gestational weeks, which is consistent with a previous report stating that detection of P. aeruginosa is uncommon within this period. ${ }^{8}$ In our study, we found that $9 \%$ infants which was detected fungus within 4 weeks after birth in both GNR positive and negative groups. This suggests that our patients were very premature infants, who are more susceptible to fungal infections, which is one of their complications. We did not administer prophylactic antifungal agents.

The etiology which GNRs makes BPD worse was indicated in several previous reports. Infective microorganisms and their products can stimulate proinflammatory cytokine production; the frequency of sepsis 7 days after birth differed significantly between infants with and without BPD. ${ }^{6}$ GNRs were also more strongly associated with worsening respiratory symptoms and festering airway secretions. Lipopolysaccharide, the main component of the GNR outer membrane, causes inflammation in immature lungs and airways, which can lead to BPD development. ${ }^{20}$ Specifically, P. aeruginosa secretes various factors, such as catalase, proteases, hemolysin, and exotoxins, which cause irreversible damage to alveolar cellular membranes. ${ }^{21}$ More proinflammatory interleukins in the alveolar fluid were found in cases with GNRs in the airways than in those with GPCs in the airways. ${ }^{13}$ The results of the present study require careful interpretation. Whether eradicating GNRs will reduce the incidence of BPD remains uncertain because, our results do not clearly distinguish the effects of colonization and invasive infection in the lungs of the premature infants we investigated.

In our study, detection of GPCs in the early postnatal period was not significantly correlated with BPD development. However, several reports contradict our results. Corynebacterium spp., classified as GPCs, were associated with severe BPD in EP infants, and more likely to be present in severe BPD infants who underwent longer durations of endotracheal ventilation. ${ }^{22}$ In our study, MRSA was detected within the first month of life in all cases with airway disease. Suzumura et al showed that infection within 14 days of intubation was considered a risk factor for acquired 
subglottic stenosis in neonates intubated for $>14$ days. ${ }^{23}$ Infants under mechanical ventilation develop airway diseases due to MRSA airway infection. These are considered to be caused by endotoxins and epidermal cell differential inhibitors that interfere with tracheal epidermal repair. ${ }^{24}$

Bacteria that contribute to BPD development are not only limited to the presence of GNRs; there is a broad bacterial diversity in tracheal cultures. Evidence suggests that the gut microbiota systematically influences immunity and inflammation, including in the lungs. ${ }^{25}$ The respiratory tract was previously considered sterile. ${ }^{26}$ Advent of 16 S rRNA genetic sequencing overcame the limitations of culture-based studies. $^{27}$ Using this technique, Laumann et $\mathrm{al}^{8}$ found that postnatal dysbiosis with decreased Lactobacillus, and increased Proteobacteria was associated with BPD development in premature infants compared with that in EP infants with and without BPD. These authors also reported that microbial communities at the phylum level showed increased relative abundances of Firmicutes and decreased Proteobacteria over time in infants with BPD. Lal et al reported the opposite result in that the airway microbiome after BPD diagnosis was characterized by increased Proteobacteria and decreased Firmicutes and Fusobacteria compared with those of full-term infants. 16S rRNA sequencing has shown that reduced diversity in the airway microbiome is associated with BPD development. ${ }^{8}$ Thus, efforts are being accelerated to characterize the airway and respiratory microbiome at birth. A cross-sectional analysis of tracheal cultures 7 days after birth showed no association with BPD severity. ${ }^{28}$

Recent studies have reported little regarding host immune responses. Ryan et al reported that RNA sequencing and 16S rRNA gene sequencing to profile gene expression in the blood and fecal microbiotas of preterm infants with or without BPD revealed that the changing microbiota systemically influences immune gene expression. ${ }^{29}$ These data suggest that changes in the gut microbiota may systematically influence immune gene expression.

This study had several limitations. First, this was a singlecenter retrospective study without predefined protocols. However, using data from a bacteriological laboratory enabled collecting precise microbiome data from all eligible EP infants with no loss to follow-up. Second, supplementary oxygen at discharge did not significantly differ between the GNR-positive and negative groups, possibly because the attending physicians lacked objective measures, such as oxygen reduction tests, to measure the need for oxygen administration before hospital discharge. Our data also showed that the GNR-positive group had significantly fewer PMA weeks at discharge, and these infants required longer durations of ventilatory management and supplementary oxygen, which may explain why durations of oxygen supplementation did not differ at discharge. Third, the days when mechanical ventilation was used were not calculated as a covariant. The longer infants were kept on a ventilator, the more likely tracheal aspirates were collected and bacteria were detected, and this increased the risk of developing BPD. In this study, the GNR-positive group had a longer mechanical ventilation time and supplementary oxygen time than the GNR-negative group, which was a different outcome from the GPC group. If a longer duration of ventilator use was a risk for developing BPD, the same result would have been expected for the GPC group, but as it did not, we excluded it as a covariant in the present study. Finally, our study only examined the tracheal microbiome via Gramstaining and did not include Ureaplasma spp. Ureaplasma spp. are significantly associated with pulmonary short- and long-term morbidity despite treatment with macrolide antibiotics. ${ }^{30}$ Ureaplasma spp. are susceptible to desiccation and temperature change and cannot be Gram-stained because they lack cell walls and cannot grow in normal media; thus, they are difficult to separate and culture. At our institution, detection of Ureaplasma spp. in infants with severe BPD was investigated selectively in cases where the mother likely had chorioamnionitis, but the data were insufficient to examine the BPD severity. In the future, we should examine Gramstained bacteria while systematically examining their relevance in the presence or absence of Ureaplasma spp.

The main strength of this study is retrospective collection of tracheal aspirate samples obtained at regular intervals during first month of life. Using that data, the association between BPD and endotracheal cultures has been shown. In the future, the use of molecular detection techniques instead of cultures can identify previously unrecognized organisms that are difficult to culture, and quantify the absolute and relative abundance of pathogenic species.

\section{Conclusion}

In this retrospective cohort study, Gram-negative bacteria in tracheal cultures were associated with BPD development within 28 days after birth for infants aged less than 26 gestational weeks. These findings were noted in the context of widespread use of broad spectrum antibiotics for 72 hours and routine saline instillation for suctioning and sample collection.

Funding

None.

Conflict of Interest

None declared.

\section{Acknowledgments}

The authors thank Drs. Sayaka Shimada, Masashi Hotta, Yasuka Kimoto, Takayuki Iwaibara, Misuzu Yoshida, and the medical staff working in NICU at OWCH for their support during this study.

\section{References}

1 Hirata K, Nishihara M, Kimura T, et al. Longitudinal impairment of lung function in school-age children with extremely low birth weights. Pediatr Pulmonol 2017;52(06):779-786

2 Hirata K, Nishihara M, Shiraishi J, Hirano S, Kitajima H, Fujimura M. Perinatal factors associated with long-term respiratory sequelae in ELBW. Arch Dis Child Fetal Neonatal Ed 2014; 0:F1-F6 
3 Miyake F, Ito M, Minami H, Tamura M, Namba F. Management of bronchopulmonary dysplasia in Japan: a 10-year nationwide survey. Pediatr Neonatol 2020;61(03):272-278

4 Fujimura M, Takeuchi T, Kitajima H, Nakayama M. Chorioamnionitis and serum IgM in Wilson-Mikity syndrome. Arch Dis Child 1989;64(10 Spec No):1379-1383

5 Jobe AH, Bancalari E. Bronchopulmonary dysplasia. Am J Respir Crit Care Med 2001;163(07):1723-1729

6 Stoll BJ, Hansen NI, Adams-Chapman I, et al; National Institute of Child Health and Human Development Neonatal Research Network. Neurodevelopmental and growth impairment among extremely low-birth-weight infants with neonatal infection. JAMA 2004;292(19):2357-2365

7 Bruce MC, Schuyler M, Martin RJ, Starcher BC, Tomashefski JF Jr, Wedig KE. Risk factors for the degradation of lung elastic fibers in the ventilated neonate. Implications for impaired lung development in bronchopulmonary dysplasia. Am Rev Respir Dis 1992; 146(01):204-212

8 Lohmann P, Luna RA, Hollister EB, et al. The airway microbiome of intubated premature infants: characteristics and changes that predict the development of bronchopulmonary dysplasia. Pediatr Res 2014;76(03):294-301

9 Mourani PM, Harris JK, Sontag MK, Robertson CE, Abman SH. Molecular identification of bacteria in tracheal aspirate fluid from mechanically ventilated preterm infants. PLoS One 2011;6(10): e25959

10 Lal CV, Travers C, Aghai ZH, et al. The airway microbiome at birth. Sci Rep 2016;6:31023

11 Lowe J, Watkins WJ, Edwards MO, et al. Association between pulmonary ureaplasma colonization and bronchopulmonary dysplasia in preterm infants: updated systematic review and metaanalysis. Pediatr Infect Dis J 2014;33(07):697-702

12 Cordero L, Sananes M, Dedhiya P, Ayers LW. Purulence and gramnegative bacilli in tracheal aspirates of mechanically ventilated very low birth weight infants. J Perinatol 2001;21(06):376-381

13 Beeton ML, Maxwell NC, Davies PL, et al. Role of pulmonary infection in the development of chronic lung disease of prematurity. Eur Respir J 2011;37(06):1424-1430

14 Itabashi K, Miura F, Uehara R, Nakamura Y. New Japanese neonatal anthropometric charts for gestational age at birth. Pediatrics international: official journal of the Japan Pediatric Society 2014;56(05):702-708

15 Bell MJ, Ternberg JL, Feigin RD, et al. Neonatal necrotizing enterocolitis. Therapeutic decisions based upon clinical staging. Ann Surg 1978;187(01):1-7

16 Papile LA, Burstein J, Burstein R, Koffler H. Incidence and evolution of subependymal and intraventricular hemorrhage: a study of infants with birth weights less than 1,500 gm. J Pediatr 1978;92 (04):529-534

17 Tramper J. The Association between positive tracheal aspirate cultures and adverse pulmonary outcomes in preterm infants with severe BPD. Am J Perinatol 2017;34:96-104

18 Cordero L, Ayers LW, Davis K. Neonatal airway colonization with gram-negative bacilli: association with severity of bronchopulmonary dysplasia. Pediatr Infect Dis J 1997;16(01):18-23

19 Groneck P, Schmale J, Soditt V. Bronchoalvelar inflammation following airway infection in preterm infants with chronic lung disease. Peddiatric pulmonology 2001;31:331-338

20 Kramer BW, Kramer S, Ikegami M, Jobe AH. Injury, inflammation, and remodeling in fetal sheep lung after intra-amniotic endotoxin. Am J Physiol Lung Cell Mol Physiol 2002;283(02):L452-L459

21 Sadikot RT, Blackwell TS, Christman JW, Prince AS. Pathogen-host interactions in Pseudomonas aeruginosa pneumonia. Am J Respir Crit Care Med 2005;171(11):1209-1223

22 Imamura T, Sato M, Go H, et al. The microbiome of the lower respiratory tract in premature infants with and without severe bronchopulmonary dysplasia. Am J Perinatol 2017;34(01):80-87

23 Suzumura H, Nitta A, Tanaka G, Kuwashima S, Hirabayashi H. Role of infection in the development of acquired subglottic stenosis in neonates with prolonged intubation. Pediatrics international: official journal of the Japan Pediatric Society 2000;42(05):508-513

24 Yamada Y, Sugai M, Woo M, Nishida N, Sugimoto T. Acquired subglottic stenosis caused by methicillin resistant Staphylococcus aureus that produce epidermal cell differentiation inhibitor. Arch Dis Child Fetal Neonatal Ed 2001;84(01):F38-F39

25 Budden KF, Gellatly SL, Wood DL, et al. Emerging pathogenic links between microbiota and the gut-lung axis. Nat Rev Microbiol 2017;15(01):55-63

26 Dickson RP, Huffnagle GB. The lung microbiome: new principles for respiratory bacteriology in health and disease. PLoS Pathog 2015;11(07):e1004923

27 Beck JM. ABCs of the lung microbiome. Ann Am Thorac Soc 2014; 11(Suppl 1):S3-S6

28 Wagner BD, Sontag MK, Harris JK, et al. Airway microbial community turnover differs by BPD severity in ventilated preterm infants. PLoS One 2017;12(01):e0170120

29 Ryan FJ, Drew DP, Douglas C, et al. Changes in the composition of the gut microbiota and the blood transcriptome in preterm infants at less than 29 weeks gestation diagnosed with bronchopulmonary dysplasia. mSystems 2019;4(05):1-17

30 Resch B, Gutmann C, Reiterer F, Luxner J, Urlesberger B. Neonatal ureaplasma urealyticum colonization increases pulmonary and cerebral morbidity despite treatment with macrolide antibiotics. Infection 2016;44(03):323-327 